\title{
Cost-effectiveness of full-course oral levofloxacin in severe community-acquired pneumonia
}

\author{
J-B. Wasserfallen*, V. Erard", A. Cometta ", T. Calandra\#, O. Lamy*
}

Cost-effectiveness of full-course oral levofloxacin in severe community-acquired pneumonia. J-B. Wasserfallen, V. Erard, A. Cometta, T. Calandra, O. Lamy. (C) ERS Journals Ltd 2004.

ABSTRACT: Oral levofloxacin is as efficient as sequential antibiotic treatment in community-acquired pneumonia (CAP). The current authors assessed whether oral levofloxacin treatment of patients with severe CAP, followed-up for 30 days, would save money.

Over a 12-month period, 129 hospitalised patients with severe non-intensive care unit CAP were randomly assigned to receive either oral levofloxacin or sequential antibiotic treatment. Direct and indirect costs were compared over a 30-day period from several perspectives.

CAP resolved in 71 out of 77 oral levofloxacin $(92 \%)$ and in 34 out of 37 sequential antibiotic treatment patients $(92 \%)$. Patients' characteristics, treatment duration, hospital length of stay and mortality were similar in both groups. Drug acquisition costs were 1.7-times smaller in oral levofloxacin patients, who were less often transferred to rehabilitation centres, but they used more physicians' visits during follow-up and their total costs were lower. As only a minority of patients was still active, inability to work and, hence, indirect costs were similar in both groups.

In this study, oral levofloxacin for severe non-intensive care unit community-acquired pneumonia was equally effective as sequential antibiotic treatment, but did not lead to major costs savings except for drug acquisition costs. External factors linked with patients' characteristics and/or medical practice are likely to play a role and should be addressed.

Eur Respir J 2004; 24: 644-648.
Services of *Medicine A and ${ }^{\#}$ Infectious Diseases, Dept of Internal Medicine, University Hospital, Lausanne, and "Service of Medicine, Centre Hospitalier YverdonChamblon, Yverdon, Switzerland.

Correspondence: J-B. Wasserfallen

Service of Medicine A

University Hospital (CHUV)

CH-1011 Lausanne

Switzerland

Fax: 41213141818

E-mail: Jean-Blaise.Wasserfallen@chuv.hospvd.ch

Keywords: Community-acquired pneumonia cost-effectiveness

economics

fluoroquinolones

oral therapy

Received: December 292003

Accepted after revision: May 202004
Community-acquired pneumonia (CAP) is a common condition especially in elderly patients [1], with a significant mortality ranging from $1 \%$ in ambulatory patients to $36.5 \%$ in intensive care patients [2]. As hospitalisations are frequent and account for $90 \%$ of total costs of treatment, costs for healthcare services are important $[3,4]$.

It is, therefore, no surprise that different measures have been studied to decrease practice variation in CAP treatment. Prediction rules have been developed to identify low-risk patients [5] who could be treated as outpatients. Different guidelines were issued about the assessment and treatment of such patients and were recently updated [6]. The duration of $i$.v. antibiotic treatment has been evaluated and an early switch to oral therapy has been advocated [7]. Both measures were shown to decrease the length of stay and, hence, the costs of hospitalisation [1, 7]. Furthermore, in-hospital observation of low-risk pneumonia patients after conversion from parenteral to oral antimicrobial therapy did not provide clinical benefit [8].

Fluoroquinolones have an excellent intestinal absorption, which makes $i$.v. administration no longer absolutely necessary. A recent review of 10 studies [9] showed that they were as effective and safe as traditional regimens. As the price of fluoroquinolones is intermediate between i.v. and oral formulations of currently used antibiotics, their use could save money $[10,11]$.

For hospitals, adoption of a global payment system according to all-patient diagnoses-related groups is an important incentive factor for the optimal use of fixed resources. In this setting, fluoroquinolones can be a very interesting kind of drug. From a societal perspective, however, the additional cost of the oral formulation can increase the whole cost of treatment. The effectiveness and safety of a full course of oral levofloxacin versus conventional sequential antibiotic therapy in patients with severe CAP requiring hospitalisation and follow-up for 30 days have been previously assessed in a clinical prospective trial [12]. However, this paper assesses whether oral levofloxacin treatment leads to direct and indirect costs savings from the perspective of a provider, payer and society.

\section{Patients and methods}

\section{Study population}

Patient selection criteria have been described in detail elsewhere [12]. In short, all consecutive adult patients requiring hospitalisation for a primary diagnosis of CAP were eligible for enrolment. Exclusion criteria included admission to an intensive care unit (ICU), high likelihood to be discharged from hospital within $24 \mathrm{~h}$ of admission, previous hospitalisation or stay in a nursing home within 10 days and 4 weeks of admission, respectively, and inability to take oral medication. 


\section{Study design and antimicrobial therapy}

The study was a prospective, randomised, controlled trial conducted in two Swiss medical centres: 1) a 900-bed teaching hospital serving both as a city hospital and as a tertiary reference centre providing services to an area of $>500,000$ inhabitants (Centre Hospitalier Universitaire Vaudois, Lausanne, Switzerland); and 2) a 160-bed community hospital (Centre Hospitalier Yverdon-Chamblon, Yverdon-les-Bains, Switzerland). The study was approved by the ethics committees of both institutions and written informed consent was obtained from each patient or legal guardian. Patients were randomised in a 2:1 ratio to a full course of oral levofloxacin (500 $\mathrm{mg}$ b.i.d.) or an i.v.-to-oral sequential therapy regimen consisting of $i$.v. ceftriaxone $(2 \mathrm{~g} q . d$. $)$ with or without concomitant i.v. or oral clarithromycin $(500 \mathrm{mg}$ b.i.d.), followed by an oral antibiotic therapy chosen by the physician in charge according to treatment guidelines for patients with CAP in use at the participating institutions. The detailed study protocol has been published elsewhere [12].

\section{Economic data}

Only patients with complete cost data were included in this economic study. Cost data used specific hospital data from 2000, as displayed in table 1. From a hospital perspective, marginal costs were computed from the running costs of the different wards divided by the observed number of patientdays in each specific unit in the year 2000. The medical, nursing, administrative and logistic cost components were singled out. Specific nursing workload, such as the insertion of an i.v. line, the administration of $i$. v. drugs and respiratory therapy, was computed according to Patient Research Nursing scores [13]. Respiratory therapist treatment was computed according to the same method as described previously. Drug acquisition costs were retrieved from the "Compendium Suisse des Médicaments", 2000 edition [14]. Rehabilitation costs were computed according to the same method as described for the hospital.

From a health insurance company perspective, hospital and rehabilitation daily charges and outpatient charges were computed from the official tariff used in Switzerland (table 1). In Switzerland's healthcare system, insurance companies pay only about half the real cost of the hospital stay (the rest being paid by the state), but the whole cost for outpatient care. The current authors did not try to compute whole costs, as overheads are difficult to single out in this healthcare system.

Finally, indirect cost linked with work inability was computed according to the Swiss standard wages of the European profession classification [15]. Both outpatient treatment and work inability were censored at day 30 after study entry.

\section{Statistical analysis}

Results were reported as mean \pm SD or SEM for normally distributed variables and as median (range) otherwise. Comparison for categorical data was performed by Chisquared test. In two-by-two situations, Yate's correction for continuity was used, whereas Fisher's exact test was used when any cell size was less than five. For continuous variables, the nonparametric Mann-Whitney U-test was used to compare the distribution between the two treatment groups. All reported significance levels are two-sided. Statistical significance was assumed at $\mathrm{p}<0.05$.
Table 1.-Costs and prices used for the economic assessment

\begin{tabular}{|c|c|c|}
\hline Type of resource & $\begin{array}{l}\text { Unit } \\
\operatorname{cost} €\end{array}$ & $\begin{array}{l}\text { Daily } \\
\text { cost } €\end{array}$ \\
\hline \multicolumn{3}{|l|}{ Provider's perspective (marginal costs) } \\
\hline \multicolumn{3}{|l|}{ Hospitalisation } \\
\hline Medical care & 43.33 & 6.84 \\
\hline Respiratory therapist & $8 \times 3.19$ & 51.12 \\
\hline \multicolumn{3}{|l|}{ Nursing care } \\
\hline Emergency dept & & 244.73 \\
\hline Ordinary ward & & 147.60 \\
\hline Intermediate care & & 333.55 \\
\hline Intensive care & & 519.49 \\
\hline \multicolumn{3}{|l|}{ Administrative tasks } \\
\hline Emergency dept & & 17.89 \\
\hline Ordinary ward & & 7.67 \\
\hline Intermediate care & & 13.42 \\
\hline Intensive care & & 19.17 \\
\hline \multicolumn{3}{|l|}{ Logistics } \\
\hline Linen & & 24.28 \\
\hline Food & & 21.73 \\
\hline \multicolumn{3}{|l|}{ Pneumonia treatment } \\
\hline Levofloxacin $2 \times 500 \mathrm{mg} \cdot \mathrm{day}^{-1}$ p.o. & 6.04 & 12.08 \\
\hline Ceftriaxone $2 \mathrm{~g} \cdot$ day $^{-1} i . v$. & 49.44 & 49.44 \\
\hline Clarithromycin $2 \times 500 \mathrm{mg} \cdot \mathrm{day}^{-1}$ p.o. & 4.01 & 8.02 \\
\hline Clarithromycin $2 \times 500 \mathrm{mg} \cdot \mathrm{day}^{-1}$ i.v. & 32.62 & 65.24 \\
\hline Infusion line insertion & $4 \times 3.39$ & 13.55 \\
\hline Infusion administration & $2 \times 3.39$ & 6.77 \\
\hline Inhalation therapy & $9 \times 3.39$ & 30.48 \\
\hline \multicolumn{3}{|l|}{ Rehabilitation centre } \\
\hline Medical care & 38.00 & 4.60 \\
\hline Nursing care & & 97.76 \\
\hline Administration & & 8.95 \\
\hline \multicolumn{3}{|l|}{ Logistics } \\
\hline Linen & & 24.28 \\
\hline Food & & 21.73 \\
\hline \multicolumn{3}{|l|}{ Payer's perspective (charges) } \\
\hline \multicolumn{3}{|l|}{ Hospital day } \\
\hline Ordinary ward & & 266.67 \\
\hline Intermediate care & & 500.00 \\
\hline Intensive care & & 1000.00 \\
\hline Rehabilitation day & & 196.67 \\
\hline \multicolumn{3}{|l|}{ Outpatient care } \\
\hline Medical visit & 40.26 & \\
\hline Respiratory therapy & 27.92 & \\
\hline Chest radiograph & 58.50 & \\
\hline \multicolumn{3}{|l|}{ Society's perspective (indirect costs) } \\
\hline \multicolumn{3}{|l|}{ Hourly wages for working patients } \\
\hline Healthcare worker & 22.88 & \\
\hline Administrative worker & 19.17 & \\
\hline Market worker & 14.76 & \\
\hline Craft worker & 19.17 & \\
\hline
\end{tabular}

Data are presented as $n$.

\section{Results}

From April 2000-April 2001, 201 patients admitted with CAP were screened, 144 were eligible for the study, 15 refused to participate and 129 were randomised. A total of 15 patients were withdrawn from the study after randomisation because of incorrect entry diagnosis $(n=10)$, loss to follow-up $(n=2)$, missing cost data $(n=2)$ or discharge from hospital within $24 \mathrm{~h}$ of admission $(n=1)$. All of the 114 remaining patients were available for analysis (77 in the levofloxacin group and 37 in the sequential therapy group).

Patients' characteristics (table 2) were comparable in the two treatment groups. In both treatment groups, $>60 \%$ of the 
Table 2. - Patients' social and clinical characteristics

\begin{tabular}{lcc}
\hline Characteristics & $\begin{array}{c}\text { Oral } \\
\text { levofloxacin }\end{array}$ & $\begin{array}{c}\text { Sequential } \\
\text { treatment }\end{array}$ \\
\hline Patients n & 77 & 37 \\
Mean age yrs & $70.7 \pm 17.1$ & $69.8 \pm 19.2$ \\
Sex & $32(42)$ & $12(32)$ \\
$\quad$ Female & $45(58)$ & $25(68)$ \\
$\quad$ Male & $15(19)$ & $6(16)$ \\
Profession & $7(9)$ & $2(5)$ \\
$\quad$ Active & $55(72)$ & $29(79)$ \\
Housekeeping & & \\
$\quad$ Retiree & $0(0)$ & $1(3)$ \\
Pneumonia Severity Index & $14(18)$ & $8(22)$ \\
I & $14(18)$ & $5(14)$ \\
II & $45(59)$ & $20(54)$ \\
III & $4(5)$ & $3(8)$ \\
IV & $57(72)$ & $27(73)$ \\
V & &
\end{tabular}

Data are expressed as $\mathrm{n}$, mean $\pm \mathrm{SD}$ and $\mathrm{n}(\%)$.

patients (72 out of 114) had a pneumonia severity index (PSI) of IV or V and $>70 \%$ of the patients ( 84 out of 114 ) had comorbidities, mostly cardiopulmonary diseases.

Outcome characteristics are displayed in table 3. Treatment duration was similar in both groups (levofloxacin mean $12.1 \pm 8.7$ days versus sequential treatment mean $13.5 \pm 3.6$ days). In the $i$.v.-to-oral sequential therapy group, 20 patients $(54 \%)$ were treated with ceftriaxone plus oral clarithromycin and 17 patients $(46 \%)$ with ceftriaxone monotherapy. Ceftriaxone was administered for a median (range) duration of 4.5 days (2-28).

The cure rate was similar ( 71 out of $77,92 \%$ ) in the levofloxacin group and in the sequential therapy group (34 out of $37,92 \%$ ). Four patients died (overall mortality $3.4 \%$ ) (levofloxacin $\mathrm{n}=1,1.3 \%$; i.v.-to-oral sequential therapy $\mathrm{n}=3$, $8.1 \%$; difference $-6.8 \%(95 \%$ CI $-16.0-2.3))$; all had a PSI of IV and were aged 79-87 yrs. Three of these deaths occurred during the hospital stay and only one (from the sequential therapy group) during the follow-up period.

The hospital length of stay was similar in both groups, but more variable in the levofloxacin group. Two patients in the levofloxacin group were clearly outliers with a length of stay of 69 and 72 days, respectively. There was no difference in the number of $i$.v. lines inserted, inhalation therapy or treatments by the respiratory therapist between the two groups.
Corresponding cost data are displayed in table 4. As usual, the cost distribution was not normally distributed and the two outliers in the levofloxacin group had a significant impact, so that, from a hospital perspective, total hospital costs were higher in the levofloxacin group. Excluding these two patients inverted the trend and the median difference between the two groups reached statistical significance (median difference $€ 650, \mathrm{p}=0.05)$. In all analyses, a highly significant difference was observed in drug-acquisition costs: sequential treatment was 1.7 times more expensive than oral levofloxacin $(95 \% \mathrm{CI}$ 22.6-307.0, $\mathrm{p}=0.023)$. Nurses' work time with drug administration was also reduced in the levofloxacin group. The proportion of patients sent to rehabilitation was slightly smaller in the oral levofloxacin group $(n=17,22 \%$ versus $n=11$, $30 \%$ ), and the mean length of stay was similar. As a consequence, the total of hospital and rehabilitation marginal costs was not different between the two groups.

The same was true when the analysis was carried out with tariffs from a health insurance perspective. However, given the smaller proportion of patients sent to rehabilitation in the levofloxacin group, the number of patients requiring physicians' visits in the 30-day follow-up was higher in the oral levofloxacin group, but this difference did not reach statistical significance. There was no difference in the number of treatments by physical therapists and chest radiographs performed during the 1-month outpatient follow-up.

As far as indirect costs were concerned, all active patients in the levofloxacin group $(n=14)$ and five out of six in the sequential treatment group were temporarily unable to work. There was no difference in the duration of work leave (table 3) and the indirect costs incurred by the two groups were not different (table 4).

\section{Discussion}

While a full course of oral levofloxacin, the latest advocated step in improving practice patterns in the treatment of CAP, brought the same benefit to the patients as standard $i$.v.-tooral sequential therapy, it did not result in obvious savings for the healthcare system in the specific setting studied, either from a provider's or a payer's perspective. In particular, the statistically significant savings in drug acquisition cost were offset by other charges linked with treating these patients. However, excluding the two patients with especially long hospital stays in the levofloxacin group lowered average hospital and rehabilitation costs in the levofloxacin group

Table 3. - Distribution of hospital resources use and post-hospital orientation and outcome

\begin{tabular}{|c|c|c|c|}
\hline Treatment characteristics & Oral levofloxacin & Sequential treatment & p-value \\
\hline Patients $\mathrm{n}$ & 77 & 37 & \\
\hline Hospital length of stay days & $9.8 \pm 11.0$ & $9.8 \pm 6.3$ & 0.181 \\
\hline Antibiotics treatment duration days & $12.1 \pm 8.7$ & $13.5 \pm 3.6$ & 0.230 \\
\hline \multicolumn{4}{|l|}{ Rehabilitation } \\
\hline Number of patients & 17(22) & 11(30) & \\
\hline Length of stay days & $25.5 \pm 14.8$ & $17.3 \pm 7.1$ & 0.074 \\
\hline \multicolumn{4}{|l|}{ Outpatient care } \\
\hline Number of patients & $76(100)$ & $35(100)$ & \\
\hline Physician visits & $1.2 \pm 1.1$ & $0.8 \pm 0.8$ & 0.078 \\
\hline Respiratory therapist visits & $0.1 \pm 0.8$ & $0.2 \pm 0.8$ & 0.459 \\
\hline Chest radiographs & $0.2 \pm 0.4$ & $0.2 \pm 0.5$ & 0.931 \\
\hline \multicolumn{4}{|l|}{ Inability to work } \\
\hline Number of patients & $15(19.5)$ & $5(13.5)$ & \\
\hline Days off work & $21.0 \pm 7.9$ & $21.6 \pm 10.2$ & 0.752 \\
\hline
\end{tabular}

Data are presented as $\mathrm{n}$, mean $\pm \mathrm{SD}$ and $\mathrm{n}(\%)$ unless otherwise stated. 
Table 4.-Distribution of the different costs of community-acquired pneumonia treatment

\begin{tabular}{lcc}
\hline Type of costs & Oral levofloxacin costs $€$ & Sequential treatment costs $€$ \\
\hline Patients n & 77 & 37 \\
Provider's perspective (marginal costs) & & $70.3 \pm 45.1$ \\
Hospitalisation & $69.6 \pm 78.9$ & $239.8 \pm 148.8$ \\
$\quad$ Physician attending & $205.5 \pm 158.7$ & $217.7 \pm 287.6$ \\
Nurses work with drugs & $250.7 \pm 499.5$ & $415.4 \pm 271.4$ \\
Physical therapist & $243.4 \pm 527.5$ & $332.6 \pm 278.3$ \\
Drug acquisition cost & $87.1 \pm 515.0$ & $82.8 \pm 43.9$ \\
$\quad$ i.v. & $156.3 \pm 107.5$ & 0.273 \\
Oral & $2717.2 \pm 5255.5$ & 0.709 \\
Hotel cost & $3486.4 \pm 6199.4$ & $2275.6 \pm 1545.8$ \\
Total hospital & $853.6 \pm 1914.7$ & $3218.7 \pm 1948.3$ \\
Rehabilitation & $4404.1 \pm 7097.3$ & $777.7 \pm 1337.1$ \\
Total marginal costs & & $4044.5 \pm 2551.6$ \\
Payer's perspective (charges) & $3644.7 \pm 8212.3$ & $<0.001$ \\
Hospital care & $1033.3 \pm 2317.8$ & 0.618 \\
Rehabilitation care & & 0.798 \\
Outpatient care & $49.4 \pm 48.0$ & 0.829 \\
Physician visits & $3.4 \pm 22.2$ & 0.766 \\
Respiratory therapist & $11.1 \pm 27.5$ & $941.4 \pm 1618.7$ \\
Chest radiograph & $64.1 \pm 66.2$ & $0.4 \pm 2155.4$ \\
Total outpatient care & $4742.2 \pm 9278.2$ & $31.8 \pm 34.9$ \\
Total charges & & $4.7 \pm 24.3$ \\
Indirect & $746.2 \pm 1883.7$ & $11.5 \pm 28.2$ \\
Patient inability to work & & $48.0 \pm 57.3$ \\
\hline
\end{tabular}

Data are presented as $\mathrm{n}$ and mean $\pm \mathrm{SD}$, unless otherwise stated.

below those observed in the sequential therapy group, but the difference was not statistically significant.

Treatment of CAP has come a long way from a full course of $i$. v. antibiotics to switch therapy and now, tentatively, to a full course of oral fluoroquinolone. Treatment with oral antibiotics helps to minimise the use of $i$.v. catheters, reducing nursing time and improving the patient's comfort and mobility, and, thus, contributing to prevent complications of $i$.v. therapy, such as phlebitis, catheter-related infections, bedsores and thrombo-embolic events.

Economic studies have assessed the full cost of CAP for healthcare systems [3, 4] and shown it to be important. Hospitalisation costs represent an important share of total costs. FINE et al. [16] found that these costs are greatest in the first 3 days after admission, with room costs amounting to $59 \%$ of the median daily costs and remaining stable throughout the hospital stay, suggesting that substantial savings would result from a 1-day decrease in the length of stay (US\$680 per patient). Using a critical pathway, including a prediction rule to assist the admission decision and guidelines for an early switch from i.v.-to-oral therapy, was shown to result in a 1.7-day reduction in the hospital length of stay (4.4 days versus 6.3 days, $\mathrm{p}=0.01$ ) and an estimated cost saving of US\$1,700 per patient [1].

Surprisingly, only two studies addressed the cost of the different antibiotic regimen used in practice [17] or proposed in guidelines [18]. Similarly, few economic comparisons were carried out for fluoroquinolones versus $\beta$-lactam or macrolide regimens in CAP. DRESSER et al. [10] examined the costeffectiveness of gatifloxacin versus ceftriaxone with a macrolid and found similar efficacy, length of stay and antibioticrelated length of stay. The cost-effectiveness ratio for gatifloxacin was US\$5,236 for each patient cured, as compared with US\$7,047 for ceftriaxone. Cost of hospitalisation was the key factor in this analysis. DRUMMOND et al. [11] assessed the costs over 21 days of sequential i.v./p.o. moxifloxacin therapy compared to i.v.lp.o. co-amoxiclav with or without clarithromycin in the patients of the
TARGET study in two different healthcare systems (Germany and France). The authors found that moxifloxacin treatment resulted in $5.3 \%$ more patients being clinically cured after 5-7 days, apyrexia achieved 1 day sooner and a 0.81 -day reduction in hospital stay, resulting in a cost saving of $€ 266$ and $€ 381$ for Germany and France, respectively.

Several factors can explain why the current authors' study found no difference in costs between the two groups. First, in these severe CAP patients not requiring treatment in an ICU, the hospital length of stay was no longer influenced by the kind of the treatment for CAP, but rather by the age of the patients, the severity of the acute disease, as well as the presence of co-morbidities, as already described by FINE et al. [5]. The need for rehabilitation in a quarter of these patients and the length of this second stay are arguments to support this influence. Similarly, the inability to work, which affected nearly all of the current authors' active patients, is a second argument in favour of this explanation.

A second factor that deserves further study may be that physicians using the full course of oral levofloxacin may have been overcautious in assessing the success of the new kind of therapy and, as a consequence, observed the patients in hospital for a longer period than was absolutely necessary. As a safeguard against a risk of underdosing due to a less than optimal absorption of oral antibiotic in these severely ill patients, levofloxacin (500 mg) was given b.i.d. instead of q.d. as is usually prescribed in patients with CAP. The high proportion of patients with a PSI of IV and V $(>60 \%)$ with co-morbidities $(>70 \%)$ and the elevated median age (77 yrs) indicate that the patients enrolled had severe non-ICU CAP [5]. This situation may change with accumulated experience and a slight decrease in length of stay may be observed in the future.

Thirdly, practice patterns have not been adapted to capture the full advantages of this new kind of treatment. Barriers to the adoption of new strategies, such as clinical practice guidelines, have already been described in CAP, where physician adherence was shown to be far from perfect [19]. 
In particular, the fact that all patients had an i.v. line inserted suggests that the admission procedures have to be changed in the future to more effectively select patients who really need $i$.v. therapy and avoid inserting a line for commodity purposes only, such as drawing blood for laboratory tests. A trend to use more procedures in teaching and in urban hospitals, as compared with rural hospitals, is well known [20] and should be addressed to reach maximal benefit.

Finally, the different results of the moxifloxacin study [11] may be due to differences in fluoroquinolone potency or to the fact that, despite excellent bioavailability of fluoroquinolones, an initial i.v. administration may be necessary to achieve early clinical improvement and earlier hospital discharge.

This study presents some obvious limitations: it involved only two hospitals and a relatively small numbers of patients, of which only a minority was still professionally active. In addition, the dose of levofloxacin (500 $\mathrm{mg} \mathrm{b.i.d.)}$ was higher than that commonly used for treating CAP, and other characteristics of the current authors' healthcare system, such as the availability of rehabilitation beds and outpatient treatment possibilities, may have influenced the pattern of care. Therefore, the ability to generalise results for both direct and indirect costs will have to be assessed in different settings.

However, the fact that the clinical outcome of these patients with community-acquired pneumonia was not different when treated with a full course of oral levofloxacin will open new perspectives in treating this disease. If these results are confirmed in other studies, they may eventually lead to interesting savings for the whole healthcare system.

\section{References}

1. Marrie JF, Lau CY, Wheeler SL, Wong CJ, Vandervoort MK, Feagan BG. A controlled trial of a critical pathway for treatment of community-acquired pneumonia. CAPITAL Study Investigators. Community-acquired pneumonia intervention trial assessing levofloxacin. JAMA 2000; 283: 749755.

2. Fine MJ, Smith MA, Carson CA, et al. Prognosis and outcomes of patients with community-acquired pneumonia. JAMA 1996; 275: 134-141.

3. Guest JF, Morris A. Community-acquired pneumonia: the annual cost to the National Health Service in the UK. Eur Respir J 1997; 10: 1530-1534.

4. Niederman MS, McCombs JS, Unger AN, Kumar A, Popovian R. The cost of treating community-acquired pneumonia. Clin Ther 1998; 20: 820-837.

5. Fine MJ, Auble TE, Yealy DM, et al. A prediction rule to identify low-risk patients with community-acquired pneumonia. New Engl J Med 1997; 336: 243-250.

6. Mandell LA, Bartlett JG, Dowell SF, File TM, Musher DM, Whitney C. Update of practice guidelines for the management of community-acquired pneumonia in immunocompetent adults. Clin Infect Dis 2003; 37: 1405-1433.

7. Ramirez JA. Switch therapy with beta-lactam/beta-lactamase inhibitors in patients with community-acquired pneumonia. Ann Pharmacother 1998; 32: S22-S26.

8. Rhew DC, Hackner D, Henderson L, Gray Ellrodt A, Weingarten SR. The clinical benefit of in-hospital observation in "low-risk" pneumonia patients after conversion from parenteral to oral antimicrobial therapy. Chest 1998; 113: $142-146$

9. Martin SJ, Sahloff EG, Close SJ. Evaluation and cost assessment of fluoroquinolones in community-acquired respiratory infections. Expert Opin Pharmacother 2002; 3 : 1251-1266.

10. Dresser LD, Niederman MS, Paladino JA. Cost-effectiveness of gatifloxacin versus ceftriaxone with a macrolide for the treatment of community-acquired pneumonia. Chest 2001; 119: $1439-1448$.

11. Drummond MF, Becker DL, Hux M, et al. An economic evaluation of sequential i.v./p.o. moxifloxacin therapy compared to i.v./p.o. co-amoxiclav with or without clarithromycin in the treatment of community-acquired pneumonia. Chest 2003; 124: 526-535.

12. Erard V, Lamy O, Bochud PY, Bille J, Cometta A, Calandra T. Full course oral levofloxacin for the treatment of hospitalized patients with community-acquired pneumonia. Eur J Clin Microbiol Infect Dis 2004; 23: 82-88.

13. Tilquin C. Un cadre conceptuel et méthodologique pour les classifications de malades selon leurs besoins en soins infirmiers [Conceptual and methodological framework for patients' classification according to their needs in nursing care]. In: Coblentz AM, Walter JR, eds. Systems science in health care. London, Taylor \& Francis, 1977; pp. 289-296.

14. Compendium Suisse des Médicaments. Morant J, Ruppanner H, eds. 21st Edn. Basel, Documed SA, 2000. www. documed.ch. Date last accessed: July 8 2004. Date last updated: July 82004.

15. International Standard Classification of Occupations (ISCO$88 \mathrm{COM})$. Warwick Institute for Employment Research. www2.warwick.ac.uk/fac/soc/ier/research/isco88/english. Date last accessed: July 8 2004. Date last updated: February 18 2004.

16. Fine MJ, Pratt HM, Obrosky DS, et al. Relation between length of hospital stay and costs of care for patients with community-acquired pneumonia. Am J Med 2000; 109: 378385.

17. Gilbert K, Gleason PP, Singer DE, et al. Variations in antimicrobial use and cost in more than 2000 patients with community-acquired pneumonia. Am J Med 1998; 104: 1727.

18. Gleason PP, Kapoor WN, Stone RA, et al. Medical outcomes and antimicrobial costs with the use of the American Thoracic Society guidelines for outpatients with community-acquired pneumonia. JAMA 1997; 278: 32-39.

19. Halm EA, Atlas SJ, Borowsky LH, et al. Understanding physician adherence with a pneumonia practice guideline: effects of patient, system, and physician factors. Arch Intern Med 2000; 160: 98-104.

20. Whittle J, Lin CJ, Lave JR, et al. Relationship of provider characteristics to outcomes, process, and costs of care for community-acquired pneumonia. Med Care 1998; 36: 977-987. 